DOI: https://doi.org/10.11144/Javeriana.upsy17-4.ppca

\title{
Propiedades psicométricas del Cuestionario de Adicción a Internet y a los Videojuegos para Adolescentes*
}

Psychometric Properties of the Internet and Videogames Addiction Questionnaire for Adolescents

Recepción: 19 Marzo 2015 | Aceptación: 09 Noviembre 2016

\author{
Nicolás Chahín-Pinzón ${ }^{\mathrm{a}}$ \\ Universidad Manuela Beltrán, Colombia \\ ORCID: http://orcid.org/0000-0001-6178-1371 \\ BlanCA LiBIA BRIÑEZ \\ Universidad de Lleida, España
}

a Autor de correspondencia. Correo electrónico:
nicocha@yahoo.com

Para citar este artículo: Chahín-Pinzón, N., \& Briñez, B. L. (2018). Propiedades psicométricas del Cuestionario de Adicción a Internet y a los Videojuegos para Adolescentes. Universitas Psychologica, 17(4), 1-13. https://doi.org/10.11144/Javeriana.upsy17-4.ppca

\section{RESUMEN}

El propósito del presente estudio fue desarrollar un instrumento que evalúe en adolescentes la adicción a internet y a los videojuegos. La muestra estuvo compuesta por 354 adolescentes $(M=13.75, D E=1.89)$. El análisis factorial confirmatorio mostró índices de ajuste aceptables $(\mathrm{CFI}=0.918, \mathrm{IFI}=0.922, \mathrm{NFI}=0.883, \mathrm{RMSEA}=0.059)$, el alfa Cronbach fue de 0.73 . De acuerdo a los resultados, el instrumento es fiable y válido para evaluar la adicción a internet y adicción a los videojuegos en adolescentes.

Palabras clave

adicción a internet; adicción a videojuegos; CAGE; adicciones no farmacológicas; adolescentes.

\begin{abstract}
The purpose of this study was to validate an instrument to assess internet and videogame addiction in adolescents. The sample consisted of 354 adolescents $(M=13.75, S D=1.89)$. The model was confirmed showing acceptable fit indices $(\mathrm{CFI}=0.918, \mathrm{IFI}=0.922$, NFI $=0.883$, RMSEA $=0.059$ ). The alpha Cronbach was 0.73. According to the results, the instrument has been shown to be a valid tool for measuring internet and video game addiction.

Keywords

internet addiction; video game addiction; CAGE; non-pharmacological addictions; adolescence.
\end{abstract}

Actualmente, las nuevas tecnologías son herramientas muy útiles en la vida cotidiana de los adolescentes. No obstante, la excesiva utilización del internet y los videojuegos ha generado numerosos problemas de diferente índole, y se ha convertido en un tema de gran interés para la comunidad científica mundial. Si bien hasta la fecha, no se encuentran tipificados como trastornos por el Diagnostic and Statistical 
Manual of Mental Disorders ([DSM-5]; American Psychiatric Association, 2013) debido a su carácter de adicción no farmacológica, está documentado que el abuso de estas tecnologías en menores de edad se encuentra asociado con diversos trastornos, tanto psicológicos como conductuales (Carbonell, Fúster, Chamarro, \& Oberst, 2012; Castellana, Sánchez-Carbonell, Graner, \& Beranuy, 2007; Durkee et al., 2012; Estévez, Bayón, de la Cruz, \& Fernández-Líria, 2009; Holtz \& Appel, 2011; Sánchez-Martínez \& Otero, 2010).

Uno de los elementos más importantes para poder determinar cuándo se está presentando la adicción o el abuso, es la elaboración de instrumentos que midan apropiadamente estas dimensiones. Aunque existen algunos para estas edades, son pocos los que han sido desarrollados o adaptados para el idioma español (Chóliz \& Marco, 2011; Gómez Salgado, Rial Boubeta, Braña Tobío, Varela Mallou, \& Barreiro Couto, 2014; Tejeiro \& Morán, 2002). En general, los instrumentos para evaluar los problemas de adicción a internet y videojuegos que se utilizan en adolescentes, provienen de escalas desarrolladas para adultos, sin que hayan tenido ningún tipo de modificación ni verificación de las propiedades psicométricas para estos sujetos (Armstrong, Phillips, \& Saling, 2000; Nichols \& Nicki, 2004).

En la búsqueda de instrumentos que puedan evaluar esta clase de adicciones, existe uno muy popular de cribado denominado CAGE (por las siglas en inglés de sus cuatro preguntas: Cut, Annoyed, Guilty, Eye), inicialmente desarrollado para evaluar problemas de alcoholismo (Ewing, 1984; Mayfield, McLeod, \& Hall, 1974). Con los años fue adaptado para medir otras adicciones en adultos, como por ejemplo: drogas, internet, móvil, etc. (Park, 2005; Thompson, 1996). Del mismo modo, el CAGE ha sido administrado a adolescentes con problemas de alcohol y otros desórdenes (Horvath, 2004; Knight, Sherritt, Harris, Gates, \& Chang, 2003; MartínezMantilla et al., 2007; Rueda-Jaimes, Pinto, Rangel, \& Camacho, 2012).

La posibilidad que ofrece el CAGE para medir diferentes tipos de adicciones, farmacológicas y no farmacológicas, pudo comprobarse cuando Pedrero et al. (2007) desarrollaron sobre la base de sus ítems, un cuestionario para adultos denominado MULTICAGE CAD 4. Este posee adecuadas propiedades psicométricas y mide 8 dimensiones: abuso/dependencia de alcohol, juego patológico, adicción a sustancias, trastornos de la alimentación, adicción a Internet, adicción a videojuegos, gasto compulsivo y adicción al sexo. El instrumento fue utilizado posteriormente en estudios con muestras de adultos, para la detección precoz de conductas adictivas (Rodríguez, Pedrero, Fernández, Gallardo, \& Sanz, 2009), detección de trastornos en el control de impulsos en pacientes con trastornos de personalidad (Martínez-González, BecoñaIglesias, \& Munera-Ramos, 2013), y para el estudio de sujetos con problemas de adicción y uso problemático del internet (Navas, Torres, Cándido, \& Perales, 2014; Pedrero, 2010).

Por otra parte, MULTICAGE CAD 4 ha sido aplicado en muestras de adolescentes para evaluar adicción a internet, videojuegos y juego patológico (Estévez, 2013; Estévez, HerreroFernández, Sarabia, \& Jauregui, 2013). Sin embargo, en estas investigaciones se utilizó la versión original para adultos sin ningún tipo de adaptación ni verificación, como es lo recomendado en estos casos (ChahínPinzón, 2015a; Chahín-Pinzón \& Briñez, 2011; Hambleton \& Zenisky, 2011; Muñiz, Elosua, \& Hambleton, 2013).

Teniendo en cuenta la importancia de tener instrumentos que midan la adicción a las nuevas tecnologías, el principal objetivo de este estudio fue el desarrollo de un cuestionario para adolescentes colombianos, compuesto por dos escalas: adicción a internet y adicción a videojuegos, a partir de las respectivas escalas del MULTICAGE CAD 4, con los siguientes objetivos específicos: a) adecuar los ítems a las características culturales, lingüísticas y de edad de la muestra; b) confirmar la estructura bifactorial; c) explorar la consistencia interna de las escalas en los grupos de edad; d) examinar las diferencias existentes en los grupos de género y edad y e) conocer la 
validez convergente del cuestionario mediante la utilización de instrumentos que evalúan agresividad e impulsividad.

\section{Método}

\section{Participantes}

La muestra para este estudio fue no probabilística y estuvo compuesta por 354 preadolescentes y adolescentes, pertenecientes a dos colegios de Bucaramanga (Colombia), de estrato socioeconómico medio: el Centro Piloto Simón Bolívar $(N=211)$ y el Gimnasio Superior $(N$ $=143)$. Las edades estuvieron entre los 11 y 18 años $(M=13.75, D E=1.89), 179$ mujeres y 175 varones. Las diferencias de edades en los dos grupos de género no resultaron significativas. La distribución de la muestra por género, rango de edades y curso puede apreciarse en la Tabla 1.

Tabla 1

Distribución de la muestra por género, edad y curso

\begin{tabular}{|c|c|c|c|c|c|c|c|c|c|c|}
\hline \multirow{2}{*}{\multicolumn{2}{|c|}{ Edad }} & \multicolumn{8}{|c|}{ Curso } & \multirow{2}{*}{ Total } \\
\hline & & 4 & 5 & 6 & 7 & 8 & 9 & 10 & 11 & \\
\hline \multirow{4}{*}{$\frac{\mathscr{0}}{\stackrel{\mathscr{J}}{\Xi}}$} & $11-12$ & 2 & 5 & 29 & 7 & 2 & 3 & - & - & 48 \\
\hline & $13-15$ & - & - & - & 4 & 21 & 40 & 20 & 5 & 90 \\
\hline & $16-18$ & - & - & - & - & 2 & 1 & 9 & 29 & 41 \\
\hline & Subtotal & 2 & 5 & 29 & 11 & 25 & 44 & 29 & 34 & 179 \\
\hline \multirow{4}{*}{ 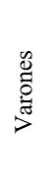 } & $11-12$ & 8 & 8 & 27 & 7 & - & 1 & - & - & 51 \\
\hline & $13-15$ & 1 & 1 & - & 9 & 29 & 24 & 23 & 5 & 92 \\
\hline & $16-18$ & - & - & - & - & - & 2 & 15 & 15 & 32 \\
\hline & Subtotal & 9 & 9 & 27 & 16 & 29 & 27 & 38 & 20 & 175 \\
\hline \multirow{4}{*}{ 氶 } & $11-12$ & 10 & 13 & 56 & 14 & 2 & 4 & - & - & 99 \\
\hline & $13-15$ & 1 & 1 & - & 13 & 50 & 64 & 43 & 10 & 182 \\
\hline & $16-18$ & - & - & - & - & 2 & 3 & 24 & 44 & 73 \\
\hline & Total & 11 & 14 & 56 & 27 & 54 & 71 & 67 & 54 & 354 \\
\hline
\end{tabular}

\section{Instrumentos}

\section{MULTICAGE CAD-4 (Pedrero et al., 2007)}

Elaborado a partir del cuestionario CAGE para detectar abuso de alcohol (Ewing, 1984). Pedrero et al. (2007) realizaron una adaptación al español para adultos sanos y adictos, desarrollando ocho escalas, cada una con 4 ítems, para un total de 32, todos con respuestas dicotómicas $(0=$ No; $1=$ Sí $)$. Dichas escalas fueron: Abuso/Dependencia de alcohol, Juego Patológico, Adicción a Sustancias, Trastornos de la Alimentación, Adicción a Internet, Adicción a Videojuegos, Gasto Compulsivo y Adicción al Sexo. Cada una de las adicciones se exploró utilizando cuatro preguntas que reproducen el esquema del CAGE, como: autopercepción del problema, percepción por parte de las personas con las que convive, sentimientos de culpa asociados y signos de abstinencia o de incapacidad para controlarse. Todas las escalas obtuvieron coeficientes $\alpha$ de Cronbach superiores a 0.7. En lo que respecta específicamente a las dos escalas que son relevantes para el presente estudio, Adicción a Internet obtuvo un $\alpha=0.82$ y Adicción a Videojuegos un $\alpha=0.79$.

A partir de los ítems de estas dos escalas, se procedió a realizar la adaptación a la población para preadolescentes y adolescentes colombianos. En esta línea, dos psicólogos con experiencia en adaptación de instrumentos revisaron la adaptación cultural y lingüística de los ítems, con el fin de que se adaptaran a las características propias de la muestra estudiada (Chahín-Pinzón, 2014; Chahín-Pinzón, 2015b; Hambleton, 1994; Hambleton, Merenda, \& Spielberger, 2005). Por ejemplo, la palabra "trabajo" se cambió por "estudio" y la palabra "ordenador" por "computador", esta última por ser más utilizada en el contexto colombiano. Posteriormente, los ítems fueron sometidos a revisión por ocho profesores con experiencia en docencia, quienes aprobaron por unanimidad los cambios realizados, obteniéndose así la versión final denominada Cuestionario de Adicción a Internet y Videojuegos para Adolescentes (CAIVA). En la Tabla 2, se encuentra la versión resultante.

Para evaluar la validez convergente se utilizaron adicionalmente los siguientes dos instrumentos: 
Cuestionario de Agresividad de Buss y Perry (AQ) (Chahín-Pinzón, Lorenzo-Seva, $\mathcal{E}$ Vigil-Colet, 2012)

Este instrumento para adolescentes está compuesto por 20 ítems, en escala tipo Likert con cinco alternativas de respuesta $(1=m u y$ rara vez; $5=$ muy frecuentemente). Posee cuatro escalas: Agresividad Física $(\alpha=0.75)$ con 7 ítems (p. ej., "Si me pegan, yo devuelvo el golpe"), Agresividad Verbal $(\alpha=0.58)$ con 4 ítems (p. ej., "Mis amigos dicen que discuto mucho"), Ira $(\alpha=0.58)$ con 4 ítems (p. ej., "Algunas veces tengo tanta rabia que me siento como si estuviera a punto de explotar") y Hostilidad $(\alpha=0.62)$ con 5 ítems (p. ej., "Algunas veces me pregunto por qué me siento tan resentido por algunas cosas").

Escala Barrat de impulsividad para niños (BIS-11c) (Chahín, Cosi, Lorenzo-Seva, Eु VigilColet, 2010)

Compuesta por 26 ítems, con escala tipo Likert con cuatro alternativas $(0=$ nunca/casi nunca; 3 = siempre/casi siempre). Posee tres escalas: Impulsividad Motora $(\alpha=0.74)$, con 13 ítems (p. ej., "Hago las cosas sin pensarlas"), Impulsividad no Planificadora $(\alpha=0.72)$ con 8 ítems (p. ej., "Organizo las cosas que hago") e Impulsividad Cognitiva $(\alpha=0.59)$ con 5 ítems (p. ej., "Decido rápidamente").

\section{Procedimiento}

Inicialmente, se solicitó autorización a los rectores y coordinadores de los respectivos colegios, luego se obtuvo el permiso de los padres. El instrumento fue aplicado colectivamente por dos psicólogos a grupos de 30 estudiantes, en los salones de clase. Los procedimientos de aplicación y manejo de la información garantizaron la confidencialidad y el anonimato. El tiempo para resolver el cuestionario osciló entre los 3 y 5 minutos.
Análisis de datos

La estructura bifactorial fue evaluada a través del análisis factorial confirmatorio (AFC). El procedimiento de estimación de máxima verosimilitud fue utilizado para la determinación de los parámetros (Byrne, 2010). Los criterios para evaluar el modelo fueron los propuestos por Hu y Bentler (1999). Como indicadores de ajuste relativo, se utilizaron: CFI (Comparative Fit Index), IFI (Incremental Fit Index) y NFI (Normative Fit Index). Y como indicadores de ajuste absoluto, el RMSEA (Root Mean Square Error of Approximation) y la razón $\chi^{2} / g l$. Aunque el consenso sobre estos índices no es general, se consideran valores aceptables para el RMSEA los inferiores a 0.08 y excelentes, los inferiores a 0.05 , y para $\chi^{2} / g l$ aceptables los menores de 3. Para el CFI, IFI y CFI son adecuados índices iguales o superiores a 0.9 (Bentler, 1990; Fan \& Sivo, 2007; $\mathrm{Hu} \&$ Bentler, 1995).

El coeficiente alfa de Cronbach fue utilizado para calcular la fiabilidad total, de las escalas, para cada uno de los grupos de edad y para toda muestra. Esto con el fin de determinar a partir de qué edades puede considerarse apropiado el instrumento. La intercorrelación de las diferentes escalas fue calculada utilizando el coeficiente $r$ de Pearson.

Se realizó un análisis de varianza en la comparación de los grupos de edad. Las diferencias de género fueron analizadas utilizando la prueba $t$ de Student para muestras independientes. En los casos en que las diferencias entre dos grupos fueron significativas, se calculó el tamaño del efecto (Cohen, 1988). La evidencia de validez convergente fue calculada utilizando el coeficiente de Pearson, al correlacionar el instrumento con el AQ y el BIS-11c. Para el análisis de los datos se utilizaron los programas estadísticos SPSS 18.0 y AMOS 18.0. 
Tabla 2

Ítems del Cuestionario de Adicción a Internet y a los Videojuegos para Adolescentes

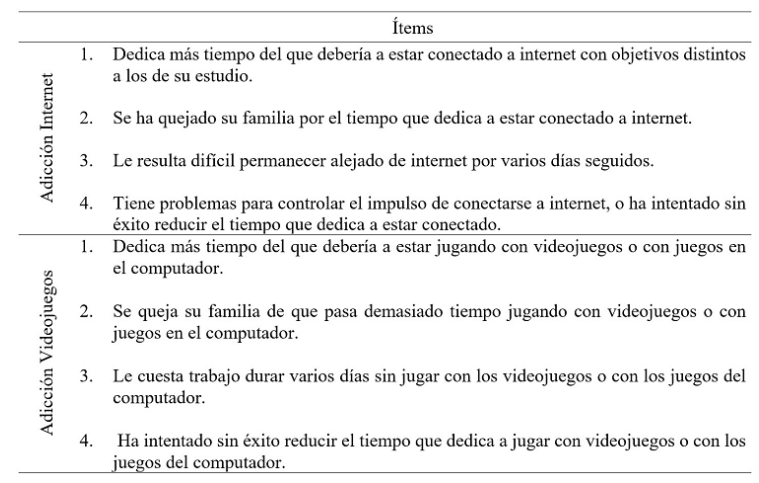

\section{Resultados}

Análisis factorial confirmatorio

Utilizando el método de máxima verosimilitud para la matriz de covarianzas, se realizó un AFC para verificar el ajuste al modelo (Arbuckle, 2009). El ajuste para el modelo bifactorial fue: $\mathrm{CFI}=0.918, \mathrm{IFI}=0.922, \mathrm{NFI}=0.883, \mathrm{RMSEA}$ $=0.059$ CI $[0.041,0.078]$ y $\chi^{2} / g l=2.78$. Los índices de ajuste relativo CFI y el IFI obtuvieron valores por encima del punto de corte de 0.9, mientras que el NFI quedó unas centésimas por debajo. Por otra parte, el RMSEA alcanzó un valor aceptable, ya que se encuentra por debajo de 0.08 , y la razón $\chi^{2} / g l$ estuvo por debajo de 3 . En la Figura 1, se puede apreciar el modelo.

Un AFC adicional se llevó a cabo para explorar la posibilidad de un modelo unifactorial. Como puede verse, los indicadores fueron bastante inferiores en comparación a los que exhibió el modelo bifactorial: $\mathrm{CFI}=0.862$, IFI $=0.867$, $\mathrm{NFI}=0.8 .29, \mathrm{RMSEA}=0.077$ CI $[0.058$, $0.093]$ y $\chi^{2} / g l=3.86$. Por tanto se descarta esta hipótesis.

\section{Figura 1}

Modelo obtenido en el análisis factorial confirmatorio.

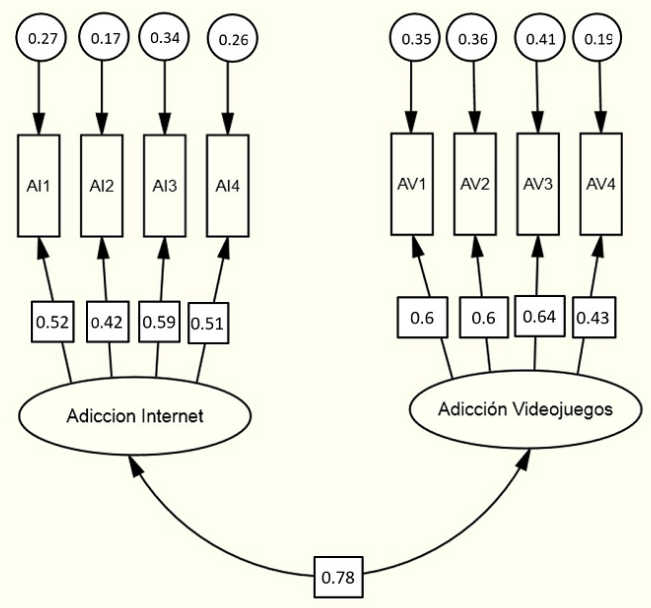

Nota. $\chi^{2}=52.44, \mathrm{gl}=19, p$-valor $=0$; RMSEA $=0.059 ; \mathrm{CFI}=0.918 ; \mathrm{IFI}=0.922 ; \mathrm{NFI}=0.883$.

\section{Estadísticos descriptivos}

La Tabla 3 presenta los valores de la media y la desviación estándar por grupos de género y edad, para la escala AI y AV.

Tabla 3

Descriptivos por género y edad

\begin{tabular}{|c|c|c|c|c|c|c|c|c|c|c|c|c|}
\hline \multirow{3}{*}{ Tipo de adicción } & \multicolumn{4}{|c|}{ Grupos por género } & \multicolumn{6}{|c|}{ Grupos por edad } & \multirow{2}{*}{\multicolumn{2}{|c|}{ Total }} \\
\hline & Muje & & Varo & & $11-12 \mathrm{a}$ & & $13-15$ & & $16-18 \mathrm{a}$ & & & \\
\hline & Media & $D E$ & Media & $D E$ & Media & $D E$ & Media & $D E$ & Media & $D E$ & Media & $D E$ \\
\hline Adicción & 1.33 & 1.29 & 1.43 & 1.16 & 1.37 & 1.23 & 1.42 & 1.21 & 1.3 & 1.27 & 1.38 & 1.23 \\
\hline Adicción Videojuegos ( $\mathrm{AV}$ ) & 0.68 & 1.07 & 1.52 & 1.28 & 1.49 & 1.26 & 1.03 & 1.21 & 0.77 & 1.18 & 1.09 & 1.25 \\
\hline Total & 2.02 & 1.9 & 2.98 & 2.15 & 2.88 & 2.2 & 2.47 & 2.07 & 2.02 & 2.04 & 2.5 & 2.12 \\
\hline
\end{tabular}

\section{Consistencia interna}

La Tabla 4 exhibe las fiabilidades obtenidas tanto para el total de la muestra como para los tres grupos de edad. Como se observa, la escala total en todos los grupos obtuvo coeficientes superiores a 0.7. Realizando una revisión un poco más detallada, se puede ver que en el grupo entre 11 y 12 años, los coeficientes para dos escalas fueron bajos. Sin embargo, estos podrían considerarse marginalmente aceptables, ya que el valor superior de sus respectivos intervalos de confianza está por encima de coeficientes considerados tradicionalmente como aceptables. 
En el grupo entre 13 y 15 años, la escala AI también obtuvo una fiabilidad baja, pero con un coeficiente marginalmente aceptable. El grupo entre 16 y 18 años fue el que exhibió mejor consistencia interna.

\section{Tabla 4}

Coeficiente de Cronbach para los tres grupos de edad

\begin{tabular}{|c|c|c|c|c|}
\hline \multirow{2}{*}{ Tipo de adicción } & \multicolumn{3}{|c|}{ Grupos por edad } & \multirow[b]{2}{*}{ Total } \\
\hline & $11-12$ & $13-15$ & $16-18$ & \\
\hline Adicción Internet (AI) & $0.55(0.39-0.67)$ & $0.56(0.44-0.65)$ & $0.66(0.52-0.77)$ & $0.56(0.49-0.63)$ \\
\hline Adicción Videojuegos (AV) & $0.55(0.39-0.68)$ & $0.67(0.58-70.4)$ & $0.78(0.69-0.85)$ & $0.67(0.62-0.72)$ \\
\hline Total & $0.71(0.61-0.79)$ & $0.71(0.65-0.77)$ & $0.76(0.67-0.83)$ & $0.73(0.68-0.76)$ \\
\hline $\begin{array}{c}\mathrm{No} \\
\mathrm{u} \\
\mathrm{O}\end{array}$ & $\begin{array}{l}\text { Interva } \\
\text { ivel de }\end{array}$ & $\begin{array}{l}\text { de conf } \\
\text { ificació }\end{array}$ & $\begin{array}{l}\text { nza con } \\
\text { del } 95\end{array}$ & \\
\hline
\end{tabular}

Diferencias entre grupos de edad y sexo

El ANOVA para los tres grupos de edad mostró que existen diferencias significativas entre las medias para la escala total $\left(F_{(2,351)}=3.53 ; p\right.$ $<0.05)$. Específicamente, en los grupos entre 11 y 12 años, y 15 y 18 años $(t=2.61, p$ $<0.05)$, presentando el grupo de menor edad puntuaciones superiores, con un tamaño del efecto $d=0.4$.

Y con respecto a la escala AV, el análisis de varianza mostró que en los grupos de edad hay diferencias $\left(F_{(2,358)}=9.24 ; p<0.001\right)$. Y al realizar las comparaciones se encontró que el grupo entre 11 y 12 años tenía puntajes superiores con respecto al de 13 y 15 años $(t=3.04, p<$ 0.005), siendo el tamaño del efecto de $\mathrm{d}=0.37$. Del mismo modo, se hallaron diferencias entre el grupo de menor edad con el de 15 y 18 años ( $t$ $=4.14, p<0.001)$, observándose un tamaño del efecto mediano $(d=0.63)$. No se encontraron diferencias en los grupos de edad para AI.

En lo referente a los grupos por género, se encontraron diferencias significativas en las medias a favor de los varones para la escala total $(t=4.39, p<0.001)(d=0.46)$. Del mismo modo en la escala AV se observó que los varones obtuvieron puntuaciones mayores $(t=6.68, p<$ 0.001 ), obteniendo esta diferencia un tamaño del efecto alto de $d=0.72$. Y en los que respecta la $\mathrm{AI}$, no se hallaron diferencias significativas.
Correlación entre escalas y validez convergente

La correlación entre la escala AI y AV fue de $r=0.44$, mientras que la correlación de las dos escalas con el total fue superior a 0.8. Para determinar la validez convergente del CAIVA se correlacionaron las escalas AI y AV con el AQ y el BIS-11c. Como se observa en la Tabla 5 , hay relaciones significativas y positivas entre las dos escalas con las cuatro dimensiones de la agresividad del AQ, con excepción de la escala ira. Hallándose los valores altos en la correlación entre agresividad física con AI $(r=0.29, p<$ $0.01)$ y AV $(r=0.33, p<0.01)$. Por su parte, la impulsividad motora fue la única de las tres escalas de impulsividad que obtuvo correlaciones significativas con AI $(r=0.3, p<0.01)$ y AV $(r$ $=0.2, p<0.01$.

\section{Tabla 5}

Correlaciones entre el Cuestionario de Adicción a Internet Videojuegos (CAIVA) con las escalas del Cuestionario de Agresividad de Buss y Perry (AQ) y la Escala de Impulsividad de Barratt para niños (BIS-11c)

\begin{tabular}{|c|c|c|c|}
\hline Escalas & AI & $\mathrm{AV}$ & Total \\
\hline Adicción Internet (AI) & - & - & - \\
\hline Adicción Videojuegos (AV) & $0.44^{* *}$ & - & \\
\hline Total escala & $0.85^{* *}$ & $0.85^{* *}$ & \\
\hline Agresividad Física & $0.29^{* *}$ & $0.33^{* *}$ & $0.37^{* *}$ \\
\hline Agresividad Verbal & $0.21^{* *}$ & $0.20^{* *}$ & $0.24^{* *}$ \\
\hline Ira & $0.17^{* *}$ & 0.06 & $0.13^{*}$ \\
\hline Hostilidad & $0.13^{*}$ & $0.15^{* *}$ & $0.17^{* *}$ \\
\hline Total Agresividad & $0.27^{* *}$ & $0.27^{* *}$ & $0.32^{* *}$ \\
\hline Impulsividad motora & $0.30^{* *}$ & $0.20^{* *}$ & $0.31^{* *}$ \\
\hline Impulsividad cognitiva & 0.005 & 0.033 & 0.028 \\
\hline Impulsividad no planificada & 0.103 & 0.058 & 0.098 \\
\hline
\end{tabular}

\section{Discusión}

El presente estudio examinó la estructura factorial y las propiedades psicométricas del Cuestionario de Adicción a Internet y Videojuegos para Adolescentes (CAIVA) en 
una muestra colombiana de adolescentes. La estructura fue confirmada mediante el AFC, encontrándose que los indicadores de ajuste absoluto y relativo, informan un aceptable ajuste al modelo bifactorial (CFI $=0.918$, IFI $=0.922$, $\left.\mathrm{NFI}=0.883, \mathrm{RMSEA}=0.059), \chi^{2} / g l=2.78\right)$.

En lo referente a la consistencia interna, el CAIVA mostró que la escala total posee coeficientes adecuados para todos los grupos de edad. Pero en el grupo de 11 a 12 años, las escalas AI y AV presentan coeficientes relativamente bajos. Y en el de 13 a 15 años, la escala AI exhibe la misma situación. Sin embargo, se ha de tener presente que en estos tres casos el límite superior de sus respectivos intervalos de confianza incluye valores que podrían ser considerados como mínimamente aceptables. Por otra parte, en el grupo entre 16 y 18 años todas las escalas funcionaron adecuadamente. Con todo, hay que tener en cuenta que a pesar de las bajas fiabilidades reportadas generalmente en niños y adolescentes, los datos obtenidos mediante autoinformes frecuentemente son mejores indicadores de su comportamiento que los respondidos por padres o profesores (Achenbach, McConaughy, \& Howell, 1987; Cosi, Canals, HernándezMartínez, \& Vigil-Colet, 2010). Es de anotar que esta tendencia a mejorar que presenta la fiabilidad a medida que incrementa la edad, en especial en la transición de la niñez a la adolescencia y la vida adulta, no es exclusiva de este estudio, y ha sido reportada en otras investigaciones (Chahín-Pinzón \& Briñez, 2015; Chahín-Pinzón et al., 2012; Santisteban \& Alvarado, 2009).

En cuanto a las diferencias en los grupos de edad, se constató que en la escala AV el grupo de menor edad obtuvo las medias más altas, y tuvo diferencias significativas con respecto a los otros dos grupos. Adicionalmente, en las comparaciones el tamaño del efecto tuvo una magnitud entre media y alta. No obstante, otros estudios sobre el comportamiento adictivo a los videojuegos no han encontrado diferencias entre los grupos de edad en niños y adolescentes (Chóliz \& Marco, 2011; Tejeiro \& Morán, 2002). Y en la escala AI, si bien no hubo diferencias significativas, sí se presentaron mayores puntajes en los dos grupos de menor edad, lo cual está acorde con lo reportado por Beranuy, Chamarro, Graner y Carbonell (2009), quienes afirman que los jóvenes de menor edad tienden a tener mayores problemas con el uso de internet (Carbonell et al., 2012).

$\mathrm{Al}$ analizar las diferencias en los grupos de acuerdo al género, en la escala AI, los varones obtuvieron mayor puntaje que las mujeres, pero no se presentaron diferencias significativas. Este resultado concuerda con Estévez (2013), quien con adolescentes y utilizando la misma escala, obtuvo mayores valores en varones, sin diferencias estadísticamente significativas. Otros estudios que han utilizado diferentes instrumentos informan que el género no se encuentra relacionado con el uso problemático del internet (Carbonell et al., 2012; Beranuy et al., 2009; Estévez et al., 2009; Muñoz-Rivas, Navarro, \& Ortega, 2003), aunque señalan que los varones tienden a hacer un mayor uso de este medio (Madell \& Muncer, 2004; Meelissen \& Drent, 2008; Rodríguez et al., 2009; Sáinz \& López-Sáez, 2010).Y en lo referente a la escala AV, se presentaron diferencias significativos a favor de los varones, con un tamaño del efecto alto, corroborado por Estévez (2013), quien encontró diferencias significativas en la misma escala. En este sentido, otros investigadores señalan que, en la infancia y la adolescencia, los varones tienden a tener una mayor dependencia hacia los videojuegos (Chóliz \& Marco, 2011; Gentile, Lynch, Linder, \& Walsh, 2004; Gentile et al., 2011; Lucas \& Sherry, 2004).

Cabe resaltar que la correlación de $r=0.44$ entre AI y AV de este estudio, fue prácticamente igual a la informada para adultos españoles por Pedrero et al. (2007) con $r=0.46$ y por Rodríguez et al. (2009) con $r=0.48$, siendo en ambas investigaciones la relación más alta entre todas las escalas.

Los resultados con respecto a la validez convergente del instrumento, indican que las escalas AI y AV se encuentran relacionadas con los componentes impulsivos y agresivos de la personalidad, y los valores de los coeficientes de Pearson son similares a los informados 
por otros autores. La escala AV tuvo una correlación positiva con las cuatro escalas del AQ. Estos coeficientes poseen valores cercanos a los hallados en otras muestras de adolescentes en los que se ha relacionado el AQ con el Internet Addiction Test (IAT) (Kim, 2013; Matalinares et al., 2013; Young, 1998). Por su lado, la escala AI solo tuvo correlaciones significativas con la impulsividad motora. Contrario a lo informado aquí, en los estudios con adolescentes en los que se relacionó el BIS con la IAT, sí se hallaron correlaciones significativas en todas las escalas (Cao, Su, Liu, \& Gao, 2007; Lim, Gwak, Park, Kim, \& Choi, 2014; Su, Gao, Xiao, Cao, \& Feng, 2011).

Asimismo, la escala de AV presentó correlaciones positivas con todas las escalas del AQ, con excepción de la escala Ira, que es el componente emocional y afectivo de la agresividad. Al confrontar estos valores con otras investigaciones con adolescentes en los que se relaciona el AQ con distintos instrumentos que evalúan la adicción a los videojuegos, se observa que todas las escalas del AQ correlacionan significativamente (Kim, Namkoong, $\mathrm{Ku}$, \& Kim, 2008; Lemmens, Valkenburg, \& Peter, 2009; Odaci \& Çelik, 2013; Teng, Li, \& Liu, 2014). Y respecto a la relación entre la escala AV con el BIS, solo la impulsividad motora correlacionó significativamente, sin embargo, otros estudios con adolescentes que utilizaron instrumentos como el Problem-Video-GamePlaying, han reportado correlaciones con las tres escalas del BIS (Vargas, Rodríguez, Grau, Faus, \& Sánchez, 2014). Estos datos sugieren que las escalas AI y AV, se encuentran más asociadas con los componentes motores de la impulsividad, relacionados con actuar antes de pensar.

El presente estudio ofreció respaldo psicométrico al instrumento, no obstante, ha de tenerse en cuenta la limitación relacionada con el tipo de muestra utilizada pues no es representativa de la población, lo que limita la generalización de los hallazgos. Para futuras investigaciones, se proponen algunas recomendaciones. Primera, utilizar una muestra aleatoria que permita realizar generalizaciones para todo el país. Segunda, teniendo en cuenta que en niños y adolescentes la fiabilidad es menor que en adultos, y el número de ítems para cada escala es de solo cuatro, y que un menor número de ítems está asociado a una menor fiabilidad (Chahín-Pinzón, 2013). Una forma viable de incrementar el coeficiente alfa de Cronbach de AI y AV para los grupos de menor edad podría ser incrementar el número de ítems de las dos escalas. Lógicamente la construcción de estos ítems deberá estar enmarcada en la conceptualización original de Ewing (1984). Tercera, para incrementar la evidencia de la validez convergente, sería conveniente realizar estudios con instrumentos más directamente asociados con este tipo de adicciones como el IAT de Young (1998). Cuarta, realizar adaptaciones culturales y lingüísticas del instrumento, con el objeto de poder realizar comparaciones entre las distintas muestras (Byrne, 2008).

En conclusión, los resultados del presente estudio indican que el CAIVA es un instrumento válido y confiable para ser aplicado en muestras similares de preadolescentes y adolescentes colombianos, ofreciendo a investigadores, educadores e interesados en el tema de las adicciones no farmacológicas, un instrumento de aplicación sencilla que permite detectar fácil y rápidamente estos dos tipos de adicciones relacionados con las nuevas tecnologías. Se recomienda tener las precauciones del caso al utilizar el instrumento en edades en las que la fiabilidad de las escalas ha sido baja, en las que sería preferible utilizar para los respectivos análisis, los coeficientes totales.

\section{Referencias}

Achenbach, T. M., McConaughy, S. H., \& Howell, C. T. (1987). Child/ adolescent behavioral and emotional problems: Implications of cross-informant correlations for situational specificity. Psychological Bulletin, 101, 213-232. https:// doi.org/10.1037/0033-2909.101.2.213

American Psychiatric Association, DSM-5 Task Force. (2013). Diagnostic and statistical 
manual of mental disorders: DSM-5 ${ }^{\mathrm{TM}}\left(5 .^{\text {a }}\right.$ ed.). Arlington, VA: American Psychiatric Publishing, Inc.

Arbuckle, J. L. (2009). Amos ${ }^{\text {TM }} 18$ user's guide. Crawfordville, FL: AMOS Development Corporation.

Armstrong, L., Phillips, J. G., \& Saling, L. L. (2000). Potential determinants of heavier internet usage. International Journal of Human-Computer Studies, 53, 537-550. htt ps://doi.org/10.1006/ijhc.2000.0400

Bentler, P. M. (1990). Comparative fit indices in structural models. Psychological Bulletin, 107, 238-246. https://doi.org/10.1037/0033 $-2909.107 .2 .238$

Beranuy, M., Chamarro, A., Graner, C., \& Carbonell, X. (2009).Validación de dos escalas breves para evaluar la adicción a Internet y el abuso de móvil. Psicothema, 21 (3), 480-485. Recuperado de http://www .psicothema.com/pdf/3658.pdf

Byrne, B. M. (2008). Testing for multigroup equivalence of a measuring instrument: A walk through the process. Psichothema, 20(4), 872-882. Recuperado de http://www .psicothema.com/PDF/3569.pdf

Byrne, B. M. (2010). Structural equation modeling with AMOS: Basic concepts, applications, and programming. Mahwah, NJ: Erlbaum.

Cao, F., Su, L., Liu, T., \& Gao, X. (2007). The relationship between impulsivity and Internet addiction in a sample of Chinese adolescents. European Psychiatry, 22(7), 466-471. https://doi.org/10.1016/j.eurpsy.2 007.05 .004

Carbonell, X., Chamarro, A., Griffiths, M., Oberst, U., Cladellas, R., \& Talarn, A. (2012). Problematic Internet and cell phone use in Spanish teenagers and young students. Anales de Psicología, 28(3), 789-796. https://doi.org/10.6018/analesps. 28.3.156061

Carbonell, X., Fúster, H., Chamarro, A., \& Oberst, U. (2012). Adicción a internet y móvil: una revisión de estudios empíricos españoles. Papeles del Psicólogo, 33(2),
82-89. Recuperado de http://www.papelesd elpsicologo.es/pdf/2096.pdf

Castellana, M., Sánchez-Carbonell, X., Graner, C., \& Beranuy, M. (2007). El adolescente ante las tecnologías de la información y la comunicación: Internet, móvil y videojuegos. Papeles del Psicólogo, 28(3), 196-204. Recuperado de http://www.papel esdelpsicologo.es/pdf/1503.pdf

Chahín-Pinzón, N. (2013). Adaptación de dos instrumentos para niños colombianos: la Escala Barrat de Impulsividad (BIS-11c) y el Cuestionario de Agresividad de Buss y Perry (AQ) (Tesis doctoral inédita). Universitat Rovira i Virgili, Tarragona.

Chahín-Pinzón, N. (2014). Aspectos a tener en cuenta cuando se realiza una adaptación de test entre diferentes culturas. Psychologia: Avances de la Disciplina, 8(2), 109-112. htt ps://doi.org/10.21500/19002386.1225

Chahín-Pinzón, N. (2015a). Consideraciones y reflexiones acerca de la versión colombiana de la Escala Barratt de Impulsividad para niños BIS-11c. Psicogente, 18(34), 396-405. https://doi.org/10.17081/psico.18.34.514

Chahín-Pinzón, N. (2015b). Revisión de las características psicométricas de la Escala Barratt de Impulsividad (BIS) a través de su historia: desde sus orígenes hasta la actualidad. Psicología desde el Caribe, 32(2), 315-326. https://doi.org/10.14482/psdc.32. 2.6110

Chahín-Pinzón, N., \& Briñez, B. (2015). Propiedades psicométricas de la Escala de Ideología de Género en adolescentes colombianos. Universitas Psychologica, 14(1), 15-23. https://doi.org/10.11144/Jave riana.upsy14-1.ppei

Chahín, N., Cosi, S., Lorenzo-Seva, U., \& VigilColet, A. (2010). Stability of the factorial structure of Barrat's Impulsivity Scales for children across cultures: a comparison between Spain and Colombia. Psicothema, 22 (4), 983-989. Recuperado de http://www .psicothema.es/pdf/3830.pdf

Chahín-Pinzón, N., Lorenzo-Seva, U., \& Vigil-Colet, A. (2012). Características psicométricas de la adaptación colombiana 
del Cuestionario de Agresividad de Buss y Perry en una muestra de preadolescentes y adolescentes de Bucaramanga. Universitas Psychologica, 11(3), 979-988. Recuperado de http://revistas.javeriana.edu.co/index.p hp/revPsycho/article/view/979

Chóliz, M., \& Marco, C. (2011). Patrón de uso y dependencia de videojuegos en infancia y adolescencia. Anales de Psicología, 27(2), 418-426. Recuperado de http://revistas.um .es/analesps/article/view/123051/138311

Cohen, J. (1988). Statistical power analysis for the behavioral sciences. Nueva York: Erlbaum.

Cosi, A., Canals, J., Hernández-Martínez, C., \& Vigil-Colet, A. (2010). Parent-child agreement in SCARED and its relationship to anxiety symptoms. Journal of Anxiety Disorders, 24, 129-133. https://doi.org/10.1 016/j.janxdis.2009.09.008

Durkee, T., Kaess, M., Carli, V., Parzer, P., Wasserman, C., Floderus, B., ... Wasserman, D. (2012). Prevalence of pathological internet use among adolescents in Europe: Demographic and social factors. Addiction, 107, 2210-2222. https://doi.org/10.1111/j.13600443.2012.03946.x

Estévez, A. (2013). Juego en adolescentes: nuevas formas, mismas consecuencias. En Laespada, M. T. \& A. Estévez (Eds.), ¿Existen las adicciones sin sustancias? (pp. 53-62). Bilbao: Deusto.

Estévez, L., Bayón, C., de la Cruz, J., \& Fernández-Líria, A. (2009). Uso y abuso de Internet en adolescentes. En E. Echeburúa, F. J. Labrador \& E. Becoña (Eds.), Adicción a las nuevas tecnologías en adolescentes y jóvenes (pp. 101-130). Madrid: Pirámide.

Estévez, A., Herrero-Fernández, D., Sarabia, I., \& Jauregui, P. (2013). The impulsivity and sensation-seeking mediators of the psychological consequences of pathological gambling in adolescence. Journal of Gambling Studies, 31(1), 91-103. https://doi .org/10.1007/s10899-013-9419-0

Ewing, J. A. (1984). Detecting alcoholism: The CAGE questionnaire. Jama, 252(14),
1905-1907. https://doi.org/10.1001/jama.1 984.03350140051025

Fan, X., \& Sivo, S. A. (2007). Sensitivity of fit indices to model misspecification and model types. Multivariate Behavioral Research, 42, 509-529. https://doi.org/10.10 80/00273170701382864

Gentile, D. A., Choo, H., Liau, A., Sim, T., Li, D., Fung, D., \& Khoo, A. (2011). Pathological video game use among youths: a twoyear longitudinal study. Pediatrics, 127(2), 319-329. https://doi.org/10.1542/peds.2010 $-1353$

Gentile, D. A., Lynch, P. J., Linder, J. R., $\&$ Walsh, D. A. (2004). The effects of violent video game habits on adolescent hostility, aggressive behaviors, and school performance. Journal of Adolescence, 27(1), 5-22. https://doi.org/10.1016/j.adolescence .2003 .10 .002

Gómez Salgado, P., Rial Boubeta, A., Braña Tobío, T., Varela Mallou, J., \& Barreiro Couto, C. (2014). Evaluation and early detection of problematic Internet use in adolescents. Psicothema, 26(1), 21-26. doi: 10.7334/psicothema2013.109

Hambleton, R. K. (1994). Guidelines for adapting educational and psychological tests: A progress report. European Journal of Psychological Assessment, 10(3), 229-244.

Hambleton, R. K., Merenda, P. F., \& Spielberger, C. D. (Eds.). (2005). Adapting educational and psychological tests for cross-cultural assessment. Hillsdale, NJ: Erlbaum.

Hambleton, R. K., \& Zenisky, A. L. (2011). Translating and adapting tests for cross cultural assessments. En D. Matsumoto \& F. J. R. Van de Vijver (Eds.), Cross-cultural research methods in psychology (pp. 46-70). Nueva York: Cambridge University Press.

Holtz, P., \& Appel, M. (2011). Internet use and video gaming predict problem behavior in early adolescence. Journal of Adolescence, 34, 49-58. https://doi.org/10.1016/j.adolesc ence.2010.02.004

Horvath, C. W. (2004). Measuring television addiction. Journal of Broadcasting $\mathfrak{G}$ 
Electronic Media, 48(3), 378-398. https://do i.org/10.1207/s15506878jobem4803_3

Hu, L., \& Bentler, P. M. (1995). Evaluating model fit. En R. Hoyle (Ed.), Structural equation modelling: Concepts, issues and applications (pp. 76-99). Thousand Oaks, CA: Sage.

Hu, L., \& Bentler, P. M. (1999). Cutoff criteria for fit indexes in covariance structure analysis: Conventional criteria versus new alternatives. Structural Equation Modeling, 6, 1-55. https://doi.org/10.1080/107055199 09540118

Kim, E. J., Namkoong, K., Ku, T., \& Kim, S. J. (2008). The relationship between online game addiction and aggression, self-control and narcissistic personality traits. European Psychiatry, 23(3), 212-218. https://doi.org/1 0.1016/j.eurpsy.2007.10.010

Kim, K. (2013). Association between Internet overuse and aggression in Korean adolescents. Pediatrics International, 55, 703-709. https://doi.org/10.1111/ped.1217 1

Knight, J. R., Sherritt, L., Harris, S. K., Gates, E. C., \& Chang, G. (2003). Validity of brief alcohol screening tests among adolescents: A comparison of the AUDIT, POSIT, CAGE, and CRAFFT. Alcoholism. Clinical \& Experimental Research, 27(1), 67-73. https://doi.org/10.1111/j.1530-0277 .2003.tb02723.x

Lemmens, J. S., Valkenburg, P. M., \& Peter, J. (2009). Development and validation of a game addiction scale for adolescents. Media Psychology, 12(1), 77-95. https://doi.org/10. 1080/15213260802669458

Lim, J. A., Gwak, A. R., Park, S. M., Kim, D. J., \& Choi, J. S. (2014). P-71.The association between internet addiction and aggression/ impulsiveness in adolescents. Alcohol and Alcoholism, 49 (Suppl. 1), i67. https://doi.o rg/10.1093/alcalc/agu054.71

Lucas, K., \& Sherry, J. L. (2004). Sex differences in video game play: A communication-based explanation. Communication Research, 31(5), 499-523. h ttps://doi.org/10.1177/0093650204267930
Madell, D., \& Muncer, S. (2004). Gender differences in the use of the Internet by English secondary school children. Social Psychology of Education, 7(2), 229-251. https://doi.org/10.1023/B:SPOE. $0000018552.85903 .4 \mathrm{~d}$

Martínez-González, J. M., Becoña-Iglesias, E., \& Munera-Ramos, P. (2013). Drogodependientes vs. usuarios de salud mental con trastornos de personalidad: su relación con la calidad de vida, la psicopatología en Eje I, el ajuste psicológico y dinámica familiar. Anales de Psicología. 29(1), 48-53. doi.org/10.6018/ analesps.29.1.161871

Martínez-Mantilla, J. A., Amaya-Naranjo, W., Campillo, H. A., Rueda-Jaimes, G. E., Campo-Arias, A., \& DíazMartínez, L. A. (2007). Consumo de sustancias psicoactivas en adolescentes, Bucaramanga, Colombia. 1996-2004. Revista de Salud pública, 9(2), 215-229. Recuperado de http://www.scielo.org.co/pd f/rsap/v9n2/v9n2a06.pdf

Matalinares, M., Arenas, C., Díaz, G., Dioses, A., Yarlequé, L., Raymundo, O., ... Yaringaño, J. (2013). Adicción a la internet y agresividad en estudiantes de secundaria del Perú. Revista de Investigación en Psicología, 16(1), 75-93. Recuperado de http://revistasinvestigacion.unmsm.edu. pe/index.php/psico/article/viewFile/3920/3 144

Mayfield, D., McLeod, G., \& Hall, P. (1974). The CAGE Questionnaire: Validation of a new alcoholism screening instrument. American Journal of Psychiatry, 131, 1121-1123. https ://doi.org/10.1176/ajp.131.10.1121

Meelissen, M. R., \& Drent, M. (2008). Gender differences in computer attitudes: Does the school matter? Computers in Human Behavior, 24(3), 969-985. https://doi.org/10 $.1016 / j . c h b .2007 .03 .001$

Muñiz, J., Elosua, P., \& Hambleton, R. K. (2013). Directrices para la traducción y adaptación de los tests. Psicothema, 25(2), 151-157. htt ps://doi.org/10.7334/psicothema2013.24 
Muñoz-Rivas, M. J., Navarro, M. E., \& Ortega, N. (2003). Patrones de uso de Internet en población universitaria española. Adicciones, 15(2), 137-144. Recuperado de http://www.adicciones.es/in dex.php/adicciones/article/view/437/434

Navas, J. F., Torres, A., Cándido, A., \& Perales, J. C. (2014). ¿Nada' o 'un poco'? ¿'Mucho' o 'demasiado'? La impulsividad como marcador de gravedad en niveles problemático y no problemático de uso de alcohol e Internet. Adicciones, 26(2), 159-167. Recuperado de http://www.adicciones.es/index.php/adi cciones/article/view/19

Nichols, L. A., \& Nicki, R. (2004). Development of a psychometrically sound internet addiction scale: A preliminary step. Psychology of Addictive Behaviors, 18(4), 381-384. https://doi.org/10.1037/0893-164 X.18.4.381

Odaci, H., \& Çelik, Ç. B. (2013). Who are problematic internet users? An investigation of the correlations between problematic internet use and shyness, loneliness, narcissism, aggression and selfperception. Computers in Human Behavior, 29, 2382-2387. https://doi.org/10.1016/j.ch b.2013.05.026

Park, W. K. (2005). Mobile phone addiction. En R. Ling, \& P. E. Pedersen (Eds.), Mobile communications: Re-negotiation of the social sphere (pp. 253-272). Londres: Springer.

Pedrero Pérez, E. J. (2010). Detección de adicciones comportamentales en adictos a sustancias en tratamiento. Trastornos Adictivos, 12(1), 13-18. https://doi.org/10.1 016/S1575-0973(10)70005-2

Pedrero, E. J., Rodríguez, M. T., Gallardo, F. Fernández, M., Pérez, M., \& Chicharro, J. (2007). Validación de un instrumento para la detección de trastornos de control de impulsos y adicciones: el MULTICAGE CAD-4. Trastornos Adictivos, 9(4), 269-279. https:// doi.org/10.1016/S1575-0973(07)75656-8

Rodríguez, M. T., Pedrero, E. J., Fernández, M., Gallardo, F., \& Sanz, T. (2009). Detección precoz de conductas adictivas en atención primaria: utilización del MULTICAGE CAD-4. Atención Primaria, 41 (1), 25-32. h ttps://doi.org/10.1016/j.aprim.2008.04.004

Rueda-Jaimes, G. E., Pinto, J. L., Rangel, A. M., \& Camacho, P. A. (2012). Abuso de alcohol en niños y adolescentes escolarizados y factores asociados. Revista Colombiana de Psiquiatría, 41(2), 273-283. Recuperado de http://www.redalyc.org/pdf/806/806244 62017.pdf

Sáinz, M., \& López-Sáez, M. (2010). Gender differences in computer attitudes and the choice of technology-related occupations in a sample of secondary students in Spain. Computers $\mathcal{E}$ Education, 54(2), 578-587. https://doi.org/10.1016/j.comped u.2009.09.007

Sánchez-Martínez, M., \& Otero, A. (2010). Usos de Internet y factores asociados en adolescentes de la Comunidad de Madrid. Atención Primaria, 42, 79-85. https://doi.org /10.1016/j.aprim.2009.05.004

Santisteban, C., \& Alvarado, J. M. (2009). The Aggression Questionnaire for Spanish Preadolescents and Adolescents: AQ-PA. The Spanish Journal of Psychology, 12, 320-326. https://doi.org/10.1017/S1138741 600001712

Su, L. Y., Gao, X. P., Xiao, Z., Cao, F. L., \& Feng, Z. (2011). Relationship between junior high school students with internet addiction and its influencing factors. Chinese Journal of School Health, 10, 1188-1190. Recuperado de http://caod.oriprobe.com/articles/28704 691/Relationship between junior high sc hool_students_with internet addicti.htm

Tejeiro R., \& Morán, R. (2002). Measuring problem video game playing in adolescents. Addiction, 97(12), 1601-1606. https://doi.o $\mathrm{rg} / 10.1046 / \mathrm{j} .1360-0443.2002 .00218 . \mathrm{x}$

Teng, Z., Li, Y., \& Liu, Y. (2014). Online gaming, internet addiction, and aggression in chinese male students: The mediating role of low self-control. International Journal of Psychological Studies, 6(2), 89-97. https:// doi.org/10.5539/ijps.v6n2p89 
Thompson, S. (1996). Internet connectivity: Addiction and dependency study (Tesis doctoral inédita). Pennsylvania State University, Pensilvania.

Young, K. S. (1998). Internet addiction: The emergence of a new clinical disorder. CyberPsychology Eु Behavior, 1(3), 237-244. https://doi.org/10.1089/cpb.1998.1.237

Vargas, J. A., Rodríguez, A., Grau, T., Faus, G., \& Sánchez, M. (2014). New technology in child and adolescents dual pathology. European Psychiatry, 29(Suppl. 1), 1. [EPA-1413]. https://doi.org/10.1016/S0924 $-9338(14) 78617-1$

\section{Notas}

* Artículo de investigación. 